\title{
Application of passive infrared thermography for DIEP flap breast reconstruction
}

\author{
by G. Steenackers ${ }^{* * *}$, J. Peeters ${ }^{*}$ P.M. Parize ${ }^{* * *}$, W. Tjalma**** \\ * University of Antwerp, Op3Mech research group, Groenenborgerlaan 171, B-2020 Antwerp, Belgium, \\ gunther.steenackers@uantwerpen.be \\ ${ }^{* *}$ Vrije Universiteit Brussel (VUB), Acoustics \& Vibration Research Group (AVRG), B-1000 Brussels, Belgium \\ *** Department of Radiology, Antwerp University Hospital and University of Antwerp, Antwerp, Belgium \\ **** Gynecological Oncology Unit, Department of Obstetrics and Gynecology, Multidisciplinary Breast Clinic, \\ Antwerp University Hospital, University of Antwerp, Antwerp, Belgiumn
}

\begin{abstract}
In order to identify the exact location of the useful perforator for DIEP flap breast reconstruction, CT images are made in the pre-operative phase. The aim of this research is to check and visualize the blood flow in the flap by means of infrared thermography. In addition, infrared thermography will be applied during and post-operative phase. The results will be used in order to pinpoint the usefulness of IR thermography as an alternative for perforator mapping. The thermal images will help to decide which parts of the flap can or cannot be used for the DIEP flap reconstruction. By means of infrared thermography a blood vessel distribution of the abdominal wall will be visualized.
\end{abstract}

Keywords: Passive thermography; Breast cancer surgery; Reconstructive surgery; Medical imaging

\section{Introduction}

A current trend in treating breast cancer is to remove the breast and perform a reconstruction with the patient's own tissue, reducing the risk of recurrent cancer with $95 \%$. The reconstruction itself is a delicate operation with high risk of morbidity. This risks causes the necessary troubles (pain) for the patient but also a higher cost for society due to the extra invasive surgery. During a DIEP flap reconstructive operation, it happens that the blood flow is decreasing, causing a partial die off of the flap. This will result in necrosis and infection, involving extra hospitalization and surgery for the patient. By means of infrared thermography a blood vessel distribution of the abdominal wall can be visualized. Based on this information, the free flap on the abdominal wall can be prepared for optimized removal.

The aim of this research is to check and visualize the blood flow in the flap by means of infrared thermography. During the operation it is of vital importance that the flap stays blooded continuously. The thermal images will help to decide which parts of the flap can or cannot be used for the DIEP flap reconstruction. Currently, the only way to check this is by making use of a visual check and in the post-operative phase by making use of a Doppler measurement. The necrosis and infections become will only be identified in the post-operative phase, causing additional surgery to be performed. By making use of passive infrared thermography in the pre-operative phase but also during the reconstructive operation, it is our goal to identify the cause of necrosis and diminishing all negative side-effects [1].

It is necessary to choose a well-drained perforator to reduce the morbidity at the donor site. The plastic surgery technique DIEP flap is based on a source artery, named the deep inferior epigastric artery (DIEA). The DIEP flap receives its blood supply by only 1 or 2 direct perforators. Direct perforators are perforators with a diameter larger than $0.5 \mathrm{~mm}$. The lower abdomen are the preferred donor site for breast reconstruction. The current methods of microvascular breast reconstruction using this donor site include the free Transverse Rectus Abdominis Myocutaneous (TRAM) flap, the superficial inferior epigastric artery (SIEA) flap and the one that we discuss, the deep inferior epigastric perforator (DIEP) flap. Itoh and Arai [19] were the first to report on the use of the technique to locate these perforators in the planning of a deep inferior epigastric perforator (DIEP) flap. They called the technique "recovery-enhanced thermography".

Theuvenet et al [1] already described in 1986 a technique called thermographic assessment of perforating arteries in the preoperative planning of DIEP flap surgery, based on cold challenge analysis. In the end the perforator with the best blood supply to the skin has the biggest chance to be used in the DIEP flap operation. The images that are taken with the thermographic camera were compared with CT-scans. The effect of a cold challenge (cooling and warming) of the abdomen is examined in [2]. The skin surface temperature is influenced by underlying structures from a depth of about $2 \mathrm{~cm}$ [3]. The underlying perforators appears as hot spots on the images and the purpose is to locate these hot spots with a thermographic camera. Cooling and warming the abdomen before using the thermographic camera is to make the hotspots more visible on the images. 
Also in a study about the evaluation of DIRT as an alternative to CT [4] was in all the cases the selected perforator visualized with DIRT the most suitable. In the study "The value of dynamic infrared thermography" [11] were the additional perforators all localized within 1.5 to $2 \mathrm{~cm}$ from the selected perforator. A disadvantage of DIRT is that only the perforators going to the skin will be shown. With CTA other perforators will be shown not only those to the skin, these give more options to choose a good DIEP flap.

Thermography is both faster and easier than colour Doppler ultrasound or MRI because it shows a real time image and indicates the sites of perforators immediately. The Doppler method in experienced hands may last up to 120 minutes [5] while perforator mapping with thermography takes only 5 to 10 minutes, including the cooling time. In [4] the brightness of hotspots was related to the volume of Doppler sounds. So it is a truly valuable technique due the good correlation between thermographic and laser Doppler results [6][7].

\section{Methodology and measurement setup}

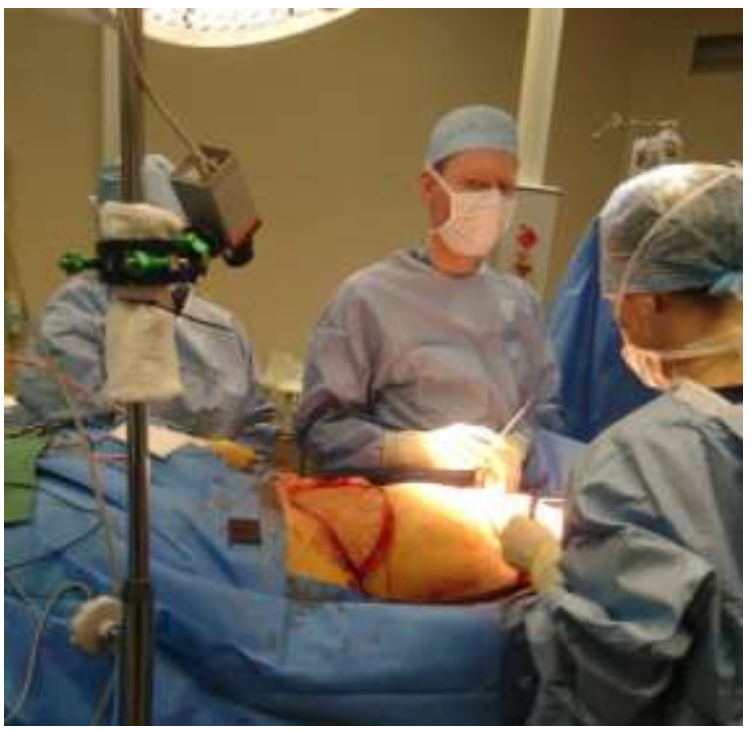

Fig. 1. Measurement setup during operation.

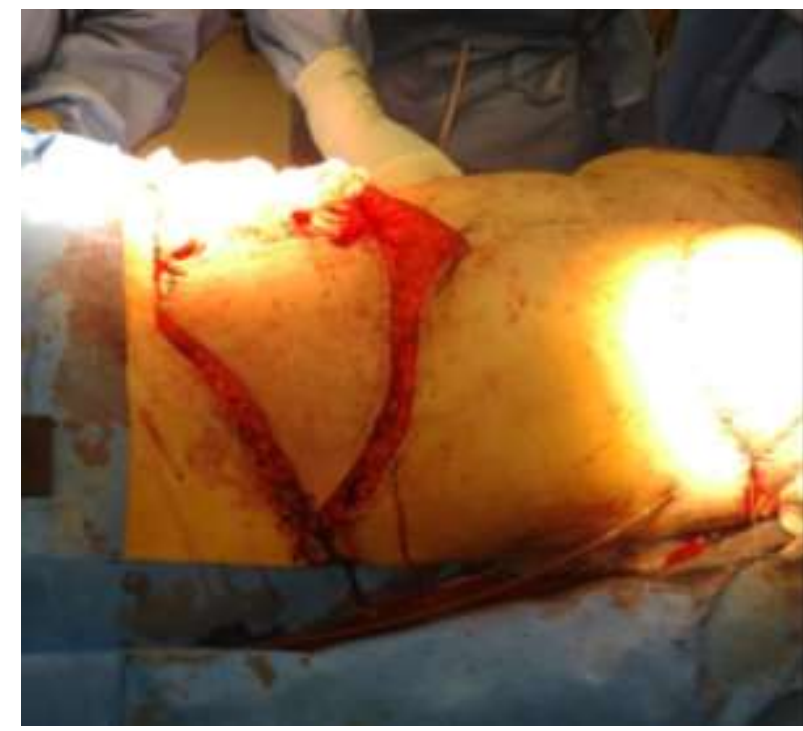

Fig. 2. Free flap during DIEP flap operation.

In order to perform an initial investigation with respect to the usage of infrared thermography during operative surgery, the researchers were invited to follow DIEP-flap reconstructive surgery and perform thermal measurements that can act as a basis for more dedicated measurements during a follow-up measurement campaign. The patient was to have a single breast reconstruction using the DIEP-flap technique. This means that both the left and right abdominal fat can be chosen to be used for the reconstruction and it can be investigated if this choice can be facilitated by making use of infrared thermography. Figure 1 shows an image of the measurement setup during operation. The microbolometer camera is placed on a tripod during operative surgery in order to measure the temperature evolution of the belly during operation. Figure 2 shows the free flap during DIEP-flap operation. At this stage, the flap is still connected with two veins, making sure the flap is still blooded and the necessary thermal measurements can be performed.

The camera used for the thermal images is a Xenics Gobi 640 microbolometer 640x480, NETD 30mK, 50Hz with 7.5$14 \mu \mathrm{m}$ spectral range. The performed measurement during DIEP flap operation include the passive thermography snapshot technique in combination with transient measurements at ca $6.25 \mathrm{~Hz}$.

The test sample is a free flap, disconnected from the abdominal wall but still connected to the body by means of two blood veins. The specimen is still in physical contact with the abdominal wall in order to keep its (body) temperature. Both blood veins will be clamped together but also separately in order to examine their influence on the bleeding of the flap during reconstructive surgery. The ambient temperature equals $20^{\circ} \mathrm{C}$ with a humidity of $35 \%$ under stable conditions and LED illumination. The measurements were performed in operating room OK14 of the Antwerp University hospital. The initial position of the chosen perforators was identified by making use a CT-scan. The position of these perforators will be double-checked during operation by making use of infrared imaging. 


\section{Results \& Discussion}

\subsection{Left flap in rest 2 veins $(A+B)$ open}

After disconnecting the left flap from the abdominal wall, the blood flow is initiated at time stamp 11:55 and it is noticeable that the temperature increases and the veins become more clear over time. This is a logic result from the blood running through both the veins $A$ and $B$. After 10 minutes, steady state conditions are achieved and the temperature profile remains stable. This is noticeable in Fig. 3 where two thermal images are shown with 10 minutes in between both measurements. It is clear that the temperature is in steady state and that there's little difference to be noticed between the two measurements. Both veins cause the flap to be blooded well.

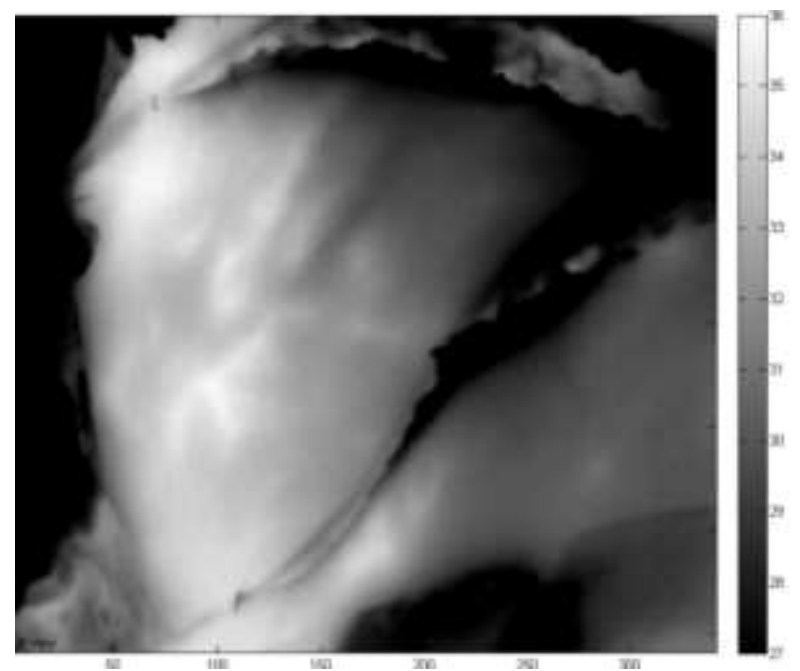

3(a): Measurement at time 11:55

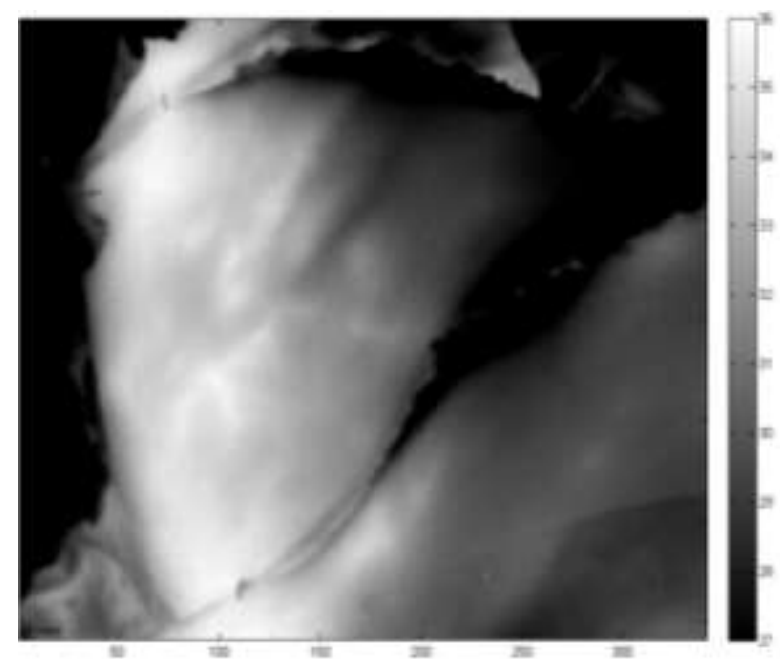

3(b): Measurement at time 12:05

Fig. 3. Thermal measurements with left flap in rest and 2 veins $(A+B)$ open 


\subsection{Left flap in rest: vein A closed and vein B open}

When opening vein $B$ but keeping vein $A$ closed, one can notice the region that is blooded by vein $B$. The more spread this region is, the more important vein $b$ is with respect to suitable perforator for the DIEP flap breast reconstruction. In Figure 4, vein $B$ is opened (clamp removed) at time instance 12:35PM and the evolution is tracked during 15 minutes. The effect of opening vein B can be visualized by looking at the thermal measurement at different time instances.

From Figure 4, one can notice that the bottom region of the flap cools down but the top region remains at a higher temperature. In addition, the veins are not clearly visible by opening vein $\mathrm{B}$. The limited predictive quality of these thermal measurements will be compared with the inverse situation when opening vein $A$ while keeping vein $\mathrm{B}$ closed.

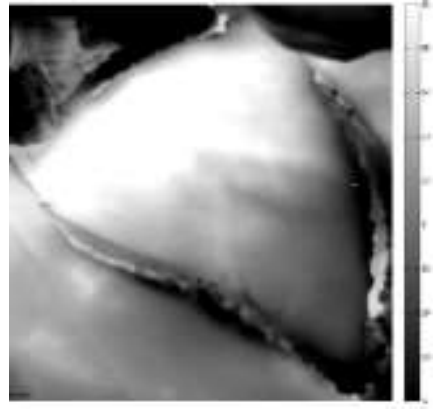

(a):Measurement at time 12:35PM

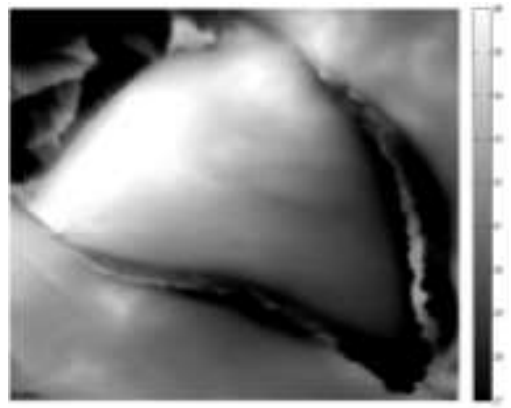

(b): Measurement at time 12:45PM

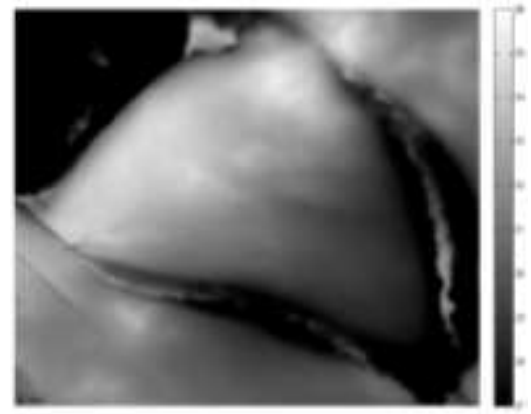

(c): Measurement at time 12:50PM

Fig. 4. Thermal measurements with left flap in rest and vein B open

\subsection{Left flap in rest: vein A open and vein B closed}

When opening vein $A$ but keeping vein $B$ closed, one can notice the region that is blooded by vein $A$. The more spread this region is, the more important vein $A$ is with respect to suitable perforator for the DIEP flap breast reconstruction. In Figure 5, vein $A$ is opened (clamp removed) at time instance 12:55PM and the evolution is tracked during 20 minutes. The effect of opening vein A can be visualized by looking at the thermal measurement at different time instances.

From Figure 5, one can notice that the bottom region of the flap cools down but the top region remains at a higher temperature, which was also detected in Figure 4. The temperature in top region slowly decreases, the temperature in largest bottom region of the flap remains more or less stable and the veins become clearly visible. On the contrary to Figure 4 with vein $B$ open, the veins are now clearly visible by opening vein $A$. From these thermal measurements, one can conclude that vein $A$ open causes a better bleed-through than with vein $B$ open. This will lead to the conclusion that vein A is a "better" perforator than vein B.

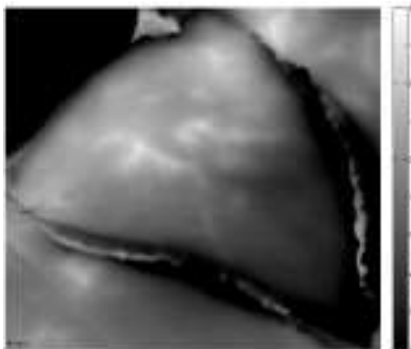

(a):Measurement at time 12:55PM

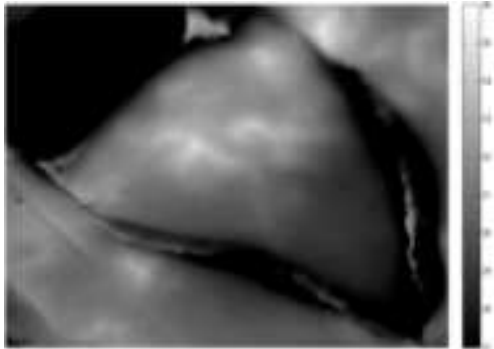

(b):Measurement at time 13:03PM

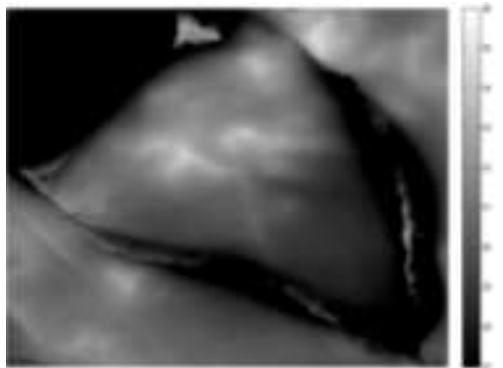

(c):Measurement at time 13:13PM

Fig. 5. Thermal measurements with left flap in rest and vein A open 


\section{Conclusion}

From the presented initial results based on a single test sample, we can conclude that infrared thermography can be a promising alternative to CTA for preoperative perforator mapping in DIEP breast reconstruction. These results are in accordance with the published results by [8]. Further investigation on more patient samples will reveal how far one can go with infrared thermography for identifying perforators in an accurate manner. Also during operation, IR thermography can identify which zone of the free flap is to be used for the reconstruction, depending on the level of bleed though in the flap.

\section{Acknowledgments}

This research was funded by the University of Antwerp and the Institute for the Promotion of Innovation by Science and Technology in Flanders (VLAIO) via support for the TETRA project, "SINT: Smart Integration of Numerical modeling and Thermal inspection," project number HBC.2017.0032. The researchers received funding from the Antwerp University IOF-council through project PSID-34924 entitled "Fast Broadband Lock-In Thermography for Fragile Structures Using System Identification."

\section{REFERENCES}

[1] W. J. Theuvenet, G. F. Koeyers, and M. H. M. Borghouts, "Thermographic Assessment of Perforating Arteries," Scand. J. Plast. Reconstr. Surg., vol. 20, no. 1, pp. 25-29, Jan. 1986.

[2] S. B. Wilson and V. A. Spence, "Dynamic thermographic imaging method for quantifying dermal perfusion: potential and limitations," Med. \{\&\} Biol. Eng. \{\&\} Comput., vol. 27, no. 5, pp. 496-501, Sep. 1989.

[3] G. Stüttgen and U. Flesch, "Dermatological Thermography," VCH Verlagsgesellschaft mbH, Weinheim, 1985

[4] S. Weum, J. B. Mercer, and L. de Weerd, "Evaluation of dynamic infrared thermography as an alternative to CT angiography for perforator mapping in breast reconstruction: A clinical study," BMC Med. Imaging, vol. 16, no. 1, 2016.

[5] R. P. Rand, M. M. Cramer, and E. D. Strandness, "Color-Flow Duplex Scanning in the Preoperative Assessment of TRAM Flap Perforators," Plast. Reconstr. Surg., vol. 93, no. 3, pp. 453-459, 1994.

[6] J. E. Francis, "Thermography as a Means of Blood Perfusion Measurement," J. Biomech. Eng., vol. 101, no. 4, p. 246, Nov. 1979.

[7] L. De Weerd, S. Weum, and J. B. Mercer, "The value of dynamic infrared thermography (DIRT) in perforator selection and planning of free DIEP flaps," Ann. Plast. Surg., vol. 63, no. 3, pp. 274-279, 2009.

[8] L. De Weerd, S. Weum, and J. B. Mercer, "Dynamic Infrared Thermography (DIRT) in the preoperative, intraoperative and postoperative phase of DIEP flap surgery," Journal of Plastic, Reconstructive and Aesthetic Surgery, vol. 65, no. 5. pp. 694-695, 2012. 